\title{
Small Group Dynamics and Extended Gas
}

\author{
F. Combes \\ DEMIRM, Observatoire de Paris, 61 Av. de l'Observatoire, F-75 014, \\ Paris, France
}

\begin{abstract}
Interactions between galaxies have spectacular effects on gas dynamics, and small galaxy groups are a privileged place to investigate them. In particular, they could test the existence of cold $\mathrm{H}_{2}$ gas as dark matter in the outer parts of galaxies. H I observations have revealed that galaxies in small groups are deficient in atomic gas, like in richer galaxy clusters such as Virgo, although in a lesser extent. Galaxy interactions could be the cause of this deficiency, stripping the gas out of galaxies and enriching the inter-cluster medium (ICM) in hot gas, which in turn strips gas through ram pressure. Alternatively, the gas present at the formation of the group could have been heated to its virial temperature, and be observed now as X-rays. The dynamical processes related to this extended gas in small galaxy groups are reviewed.
\end{abstract}

\section{Introduction}

Interaction of galaxies is a way to reveal extended gas around galaxies, that could stay cold and dark in quiescent phases. The hypothesis that the baryonic dark matter around galaxies could be cold $\mathrm{H}_{2}$ clouds has been developped in recent years, in different models. The cold clouds could be rotationally supported, orbiting in the outer parts of the galaxies in a flaring plane, associated to the $\mathrm{H} \mathrm{I}$ gas (Pfenniger et al. 1994, Pfenniger \& Combes 1994). They are distributed in a fractal structure, with the smallest fragments (the clumpuscules) having a mass $\mathrm{M} \sim 10^{-3} M_{\odot}$, a molecular density $\mathrm{n}_{\mathrm{H} 2} \sim 10^{10} \mathrm{~cm}^{-3}$, and a radius $\mathrm{R} \sim 10 \mathrm{AU}$. Alternatively, the cold $\mathrm{H}_{2}$ gas could be associated to brown dwarfs in clusters, distributed in the galactic halo, or spheroidal component (de Paolis et al. 1995; Gerhard \& Silk 1996). Their temperature would then be higher $(T=20 \mathrm{~K})$.

These clumps are seen in ESE (extreme scattering events) in front of quasars and in H I- VLBI in absorption against extended radio-sources (Fiedler et al. 1987, Walker \& Wardle 1998, Faison et al. 1998). The statistics of observations are compatible with a large number of these clumps in the Galaxy, so that the total mass could be comparable to that of the visible Milky Way.

The existence of this cold $\mathrm{H}_{2}$ gas has also been invoked to explain the extra$\gamma$ rays detected by EGRET, on board GRO (de Paolis et al. 1999, Sciama 1999) and some observational consequences have been studied (Combes \& Pfenniger 1997, Shchekinov 1999). Cooling flows in clusters could also form these clumpuscules (Ferland et al. 1994).

Let us recall that the visible matter in the Universe corresponds to only $\Omega_{v i s} \sim 0.003$, while the Big Bang nucleosynthesis implies that the baryonic 
density is $\Omega_{b} \sim 0.01 h^{-2}$, or that $0.01<\Omega_{b}<0.04$. Most of the baryons are therefore dark, and the MACHOs cannot be a significant part of them, according to the microlensing experiments.

\section{Gas Dynamics in Small Groups}

\subsection{Atomic Gas}

The most striking feature in interacting galaxy groups is the presence of large tidal tails of matter dragged out of the galaxies, huge $\mathrm{HI}$ extensions with respect to the optical systems. Yun et al. (1993) have found large quantities of $\mathrm{H} \mathrm{I}$ all around the M81/M82/NGC 3077 system, and Hibbard (1995) shows in his thesis an evolving sequence of interacting/merging galaxies, where the $\mathrm{H} I$ extensions are conspicuous. More precisely, the percentage of the total $\mathrm{HI}$ found in the tails/extensions is increasing with the merging stage, from $20 \%$ in the M81 system, to $80 \%$ in the merger remnant NGC 7252 . This does not mean that most of the gas will be expelled from interacting systems. In fact, with all probability, the gas dragged out remains bound to the system, and will rain back onto the merger remnant, after some billion years. Hibbard et al. (1994) show that the gas at the bottom of the tails in NGC 7252 is infalling.

In normal galaxies, large $\mathrm{H}$ I extents are very rare (Briggs et al. 1980, Broeils 1992, Hoffman et al. 1996). In average the gas extent is $\mathrm{R}_{\mathrm{HI}} \sim 2 \mathrm{R}_{\text {opt }}$. Only interacting systems show extended gas.

\subsection{Molecular Gas}

What is seen in the molecular phase is just the contrary: apparently large $\mathrm{H}_{2}$ concentrations pile up at the galaxy nuclei in interacting systems. Up to $50 \%$ of the dynamical mass could be under the form of molecular hydrogen in merging systems (Scoville et al. 1991), even when the the $\mathrm{CO} / \mathrm{H}_{2}$ conversion ratio is corrected to a minimum (Bryant \& Scoville 1999, Solomon et al. 1997).

In summary, the observations suggest that the $\mathrm{HI}$ gas is dragged outwards, while the $\mathrm{H}_{2}$ gas is driven inwards, to be consumed in star formation. In fact these two tracers ( $\mathrm{HI}$ and $\mathrm{CO}$ ) shed light on two aspects of the same gas component. In normal spiral galaxies, there exists a sharp cut-off in the $\mathrm{H}$ I line distribution $(21 \mathrm{~cm}$ ) (van Gorkom et al. 1991, Corbelli \& Salpeter 1993); it is interpreted as a ionizing front, due to photoionisation from the extragalactic background. $\mathrm{H}$ II is the third gas phase to take into account.

While star formation is enhanced in interacting galaxies, this is not true in groups and clusters. This is mainly due to the stripping of the outer gas acting as reservoirs of fuel for star formation. The $\mathrm{H}$ I deficiency in compact groups is on average a factor of 3 (Williams \& Rood 1987, Williams et al. 1991, Huchtmeier 1997 , Oosterloo \& Iovino 1997), and there is no significant CO deficiency or enhancement (Boselli et al. 1996, Leon et al. 1997, Verdes-Montenegro et al. 1998). The missing gas is heated by repeated interactions, and joins the coronal hot phase. ROSAT survey of 22 HCG (Ponman et al. 1996) have revealed that $75 \%$ possess a diffuse hot gas. There appears to be a correlation between X-ray and spiral galaxy fraction (Pildis et al. 1995). 


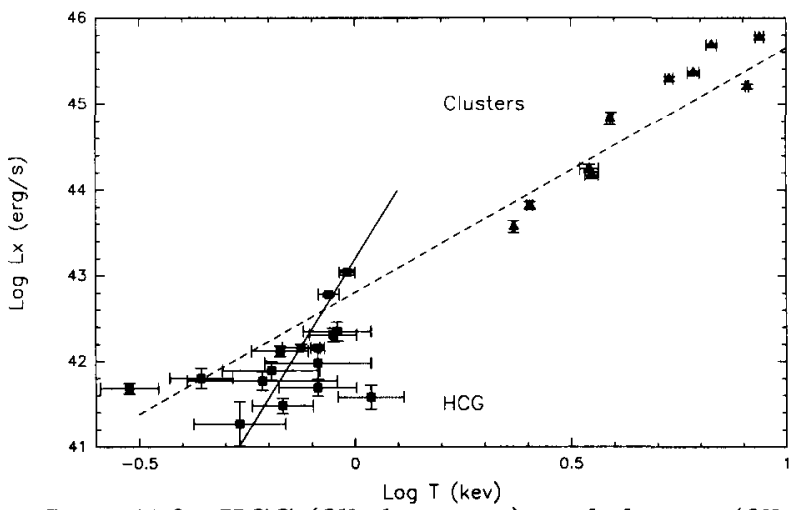

Figure 1. $\quad \mathrm{L}_{x}$ vs $\mathrm{T}$ for HCG (filled squares), and clusters (filled triangles), from Ponman et al. (1996). The dashed line is the best regression fit for clusters, and full line for HCGs.

\subsection{Hot Gas}

Hot gas and its X-ray emission are the keys to follow the fate of gas in interactions. Read \& Ponman (1998) have followed a merger sequence and found that the X-ray luminosity is enhanced by the interaction, although not as much as the FIR luminosity. Spatially, the X-ray emission is not correlated with tidal tails; the hot gas is ejected in winds, powered by the central starbursts. The classical M82-type bipolar outflow is often replaced by unipolar outflows in violent mergers.

In merger remnants (like in NGC 7252) $\mathrm{L}_{X}$ drops again. This is surprising since such remnants are bound to become classical ellipticals, which have more hot gas than spirals (e.g. Fabbiano, 1989). How is the transformation done? At the base of the tidal tails, in NGC 7252, the $\mathrm{H} \mathrm{I}$ is falling back, as revealed by its kinematics (Hibbard et al. 1994), but then stops suddenly. It is not replaced by $\mathrm{H}_{2}$ gas, that is concentrated much further in. It is likely that the returning gas is heated by shocks to form a hot gas halo. This will take a time-scale of the order of Gyrs to form a genuine elliptical.

In small groups, the hot gas is even more extended, up to $400 \mathrm{kpc}$ (Davis et al. 1995). The amount of hot gas is such that 20 to $34 \%$ of the mass is baryonic. This cannot be done by galactic winds (Ponman et al. 1996, Mulchaey et al. 1996). About $75 \%$ of compact groups have detectable X-ray emission (Ponman et al. 1996). In small groups, the hot gas metallicity is low (lower than in clusters). In the $\mathrm{L}_{X}-\mathrm{T}$ diagram, the compact groups occupy a special region, after a turn-over: they have less luminosity for a given temperature than would be expected from clusters (cf fig. 1). This could be due to the influence of galactic winds, creating a hole in the center, while winds have no influence in clusters (Ponman et al. 1996). Alternatively, the hot gas could be progressively heated, through interactions, and have not yet reached equilibrium in small groups. This would be the case if the hot gas comes from the cold molecular gas in outer parts of galaxies (Pfenniger \& Combes 1994). 


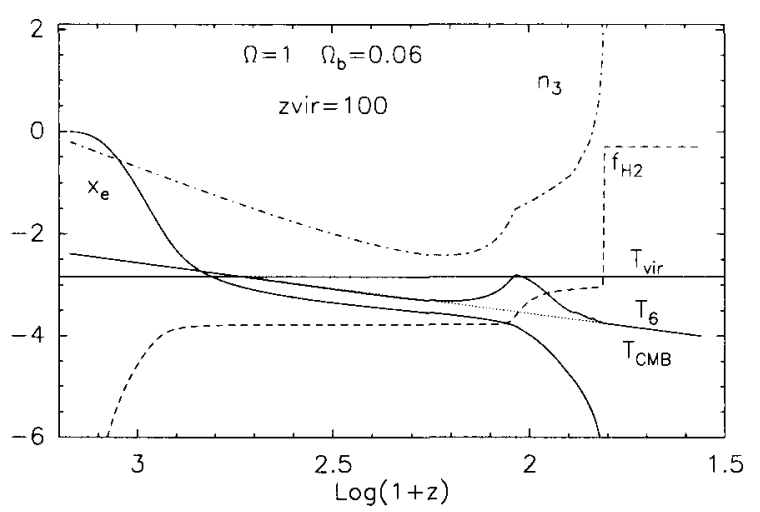

Figure 2. Evolution of temperature $\left(T_{6}\right.$ in $\left.10^{6} \mathrm{~K}\right)$, molecular fraction $\left(f_{\mathrm{H}_{2}}\right)$, ionization fraction $\left(x_{\mathrm{e}}\right)$ and density $\left(n_{3}\right.$ in $\left.10^{3} \mathrm{~cm}^{-3}\right)$ for a gas cloud after recombination, collapsing at $z_{\mathrm{vir}}=100$, with $h=0.5, \Omega=1$ and $\Omega_{\mathrm{b}}=0.06$ (from Combes \& Pfenniger 1998).

\section{Formation of Gas Clumps}

In this hypothesis, what is the fate of the gas, and when do the cold gas clumps form? After recombination $(z \sim 1500)$, the largest masses to get non-linear are of $M \sim 10^{6-8} M_{\odot}$, and the Jeans mass is $M \sim 10^{5} M_{\odot}\left(\frac{\Omega_{b}}{0.06}\right)^{-1 / 2}\left(\frac{h}{0.5}\right)^{-1}$. All masses between these two will collapse and decouple from expansion, but the structures at precisely the Jeans mass collapse first. The masses correspond to typical $z=0$ giant molecular clouds. If cooling is efficient enough $\left(\tau_{\text {cool }} \sim \tau_{\mathrm{ff}}\right)$, the collapse is quasi-isothermal, and fragmentation occurs, since the Jeans mass becomes smaller and smaller as the density increases (e.g., Hoyle 1953). Fragmentation is limited by opacity, and the smallest fragments (or clumpuscules), which are at the transition of being pressure supported, are today of the order of $10^{-3} M_{\odot}$, and their mass grows slowly, as $T^{1 / 4}$ or $(1+z)^{1 / 4}$, with redshift.

Cooling might be a problem, since primordial gas is non-metallic, with no dust grains; above $10^{4} \mathrm{~K}$ the main coolant is atomic hydrogen (by collisional excitation of $\mathrm{Ly} \alpha$ ), then vibration-rotation lines of molecular hydrogen take over until $T=200 \mathrm{~K}$, because a significant quantity of $\mathrm{H}_{2}$ molecules forms through $\mathrm{H}^{-}$ and $\mathrm{H}_{2}^{+}$. Below $200 \mathrm{~K}, \mathrm{HD}$ is then more efficient. Many groups have computed the physico-chemistry of the primordial gas, to determine the size of the first forming bound structures (Yoneyama 1972; Hutchins 1976; Carlberg 1981; Palla et al. 1983; Lepp \& Shull 1984). All of them have found that the cooling is indeed efficient, as soon as the redshift is below $z \sim 200$.

We have studied in particular the recursive formation of fragments, as expected in a fractal structure of dimension $D=1.7$ (which fixes the density as a function of sizes and masses). Cooling in such a fractal is very efficient, as shown by the temperature curve, which quickly returns back to $\mathrm{T}_{C M B}$ of the background after virialisation (cf fig. 2). Very soon, the clumps are almost entirely molecular (Combes \& Pfenniger 1998). 


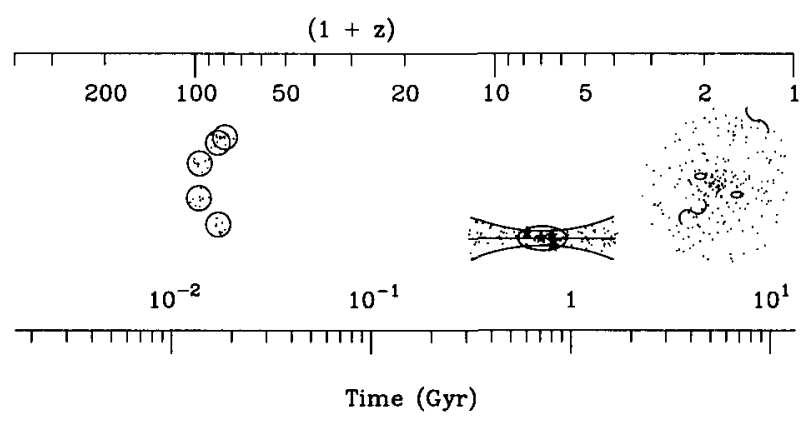

Figure 3. Schematic view of the evolution of the baryonic dark matter, under the form of cold $\mathrm{H}_{2}$ gas: formation of clumpuscules at a redshift around 100, which are progressively involved in the formation of larger structures and proto-galaxies. A typical early galaxy is shown at $z=6$, with the cold gas settling into a flaring disk, and the star formation beginning in the center, where the surface density is above threshold. Later on, when groups and clusters virialise, the gas is stripped and heated to contribute to the hot X-ray gas.

\section{Discussion}

After this efficient formation, most of the gas is not in favorable conditions for star formation. Indeed, larger potential wells have not formed yet, and the density threshold is not reached. It is therefore likely that the gas will slowly accumulate in bigger potentials to form galaxies, as sketched in fig. 3 . In the inner parts of these early galaxies, star formation can then begin. Then galaxy interactions will stir and heat the gas through shocks and gravitational perturbations.

Further, interactions accelerate the angular momentum transfer: part of the $\mathrm{HI}$ gas is dragged outwards in tails. Most of the gas is driven inwards, giving rise to huge nuclear starbursts (and maybe AGN). Galaxy evolution is highly accelerated. The cold gas that was settled around each galaxy is heated and virialised in the new common potential and might be visible through X-rays.

\section{References}

Boselli, A., Mendes De Oliveira C., Balkowski C., et al., 1996, A\&A, 314, 738

Briggs, F. H., Wolfe, A. M., Krumm, N., Salpeter, E. E., 1980 ApJ, 238, 510

Broeils, A., 1992, PhD Thesis, Groningen

Bryant, P. M., Scoville, N. Z., 1999, AJ, 117, 2632

Carlberg, R. G., 1981, MNRAS, 197, 1021

Combes, F., Pfenniger D., 1997, A\&A 327, 453

Combes, F., Pfenniger D., 1998, in "H2 in the Early Universe", ed. E. Corbelli, D. Galli, F. Palla, Mem. S. A. It. 69, 413 (astro-ph/9801319) 
Corbelli, E., Salpeter E. E., 1993, ApJ 419, 94 \& 104

Davis, D. S., Mushotzky, R. F., Mulchaey, J. S. et al. , 1995, ApJ, 444, 582

De Paolis, F., Ingrosso G., Jetzer P. et al., 1995, A\&A, 299, 647

De Paolis, F., Ingrosso, G., Jetzer, Ph., Roncadelli, M., 1999, ApJ, 510, L103

Fabbiano, G., 1989, ARAA, 27, 87

Faison, M. D., Goss W. M., Diamond P. J., Taylor G. B., 1998, AJ, 116, 2916

Ferland, G. J., Fabian, A. C., Johnstone, R. M., 1994, MNRAS, 266, 399

Fiedler, R. L., Dennison B., Johnston K., Hewish A., 1987, Nature, 326, 675

Gerhard, O., Silk, J., 1996, ApJ, 472, 34

Hibbard, J., Guhathakurta P., Van Gorkom J., Schweizer F.,1994 AJ, 107, 67

Hibbard, J. E., 1995, PhD thesis, Columbia University

Hoffman, G. L., Salpeter E. E., Farhat B. et al., 1996, ApJS, 105, 269

Hoyle, F., 1953, ApJ, 118, 513

Huchtmeier, W. K., 1997, A\&A, 325, 473

Hutchins, J. B., 1976, ApJ, 205, 103

Leon, S., Combes F., Menon K., 1998, A\&A, 330, 37

Lepp, S., Schull J. M., 1984, ApJ, 280465

Mulchaey, J. S., Davis D. S., Mushotzky R. F., Burstein D., 1996, ApJ, 456, 80

Oosterloo, T., Iovino, A., 1997, PASA, 14, 48

Palla F., Salpeter E. E., Stahler S. W., 1983, ApJ, 271, 632

Pfenniger, D., Combes F., Martinet L., 1994, A\&A, 285, 79

Pfenniger, D., Combes F., 1994, A\&A, 285, 94

Pildis, R. A., Bregman, J. N., Evrard, A. E., 1995, ApJ, 443, 514

Ponman, T., Bourner P., Ebeling H., Böhringer H., 1996, MNRAS, 283, 690

Read, A. M., Ponman T. J., 1998, MNRAS, 297, 143

Sciama, D. W., 1999, MNRAS, submitted, (astro-ph/9906159)

Shchekinov, Y. A., 1999, Astron. Rep. in press, (astro-ph/9811434)

Scoville, N. Z., Sargent, A. I., Sanders, D. B., Soifer, B. T., 1991 ApJ, 366, L5

Solomon, P. M., Downes D., Radford S. J. E., Barrett J. W., 1997, ApJ 478, 144

van Gorkom, J.H., 1991, in "Atoms, Ions and Molecules", ed. A. D. Haschik, ASP Conf. Ser. 16, 1

Verdes-Montenegro, L., Yun, M. S., Perea, J. et al.,1998, ApJ, 497, 89

Walker M., Wardle M., 1998, ApJ, 498, L125

Williams B. A., Rood H. J., 1987, ApJS, 63, 265

Williams, B. A., McMahon, P. M., Van Gorkom, J. H., 1991, AJ, 101, 1957

Yoneyama, T., 1972, PASJ, 24, 87

Yun, M. S., Ho, P. T. P., Lo, K. Y., 1993, ApJ, 411, L17 